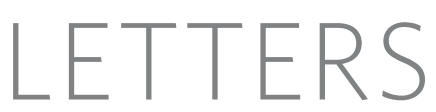

\title{
Tunable nanowire nonlinear optical probe
}

\author{
Yuri Nakayama ${ }^{1,6 \star}$, Peter J. Pauzauskie ${ }^{1,5 \star}$, Aleksandra Radenovic ${ }^{2,4 \star}$, Robert M. Onorato ${ }^{1 \star}$, Richard J. Saykally ${ }^{1,4}$, \\ Jan Liphardt ${ }^{2,3,4} \&$ Peidong Yang ${ }^{1,5}$
}

One crucial challenge for subwavelength optics has been the development of a tunable source of coherent laser radiation for use in the physical, information and biological sciences that is stable at room temperature and physiological conditions. Current advanced nearfield imaging techniques using fibre-optic scattering probes ${ }^{1,2}$ have already achieved spatial resolution down to the $20-\mathrm{nm}$ range. Recently reported far-field approaches for optical microscopy, including stimulated emission depletion ${ }^{3}$, structured illumination ${ }^{4}$, and photoactivated localization microscopy ${ }^{5}$, have enabled impressive, theoretically unlimited spatial resolution of fluorescent biomolecular complexes. Previous work with laser tweezers ${ }^{6-8}$ has suggested that optical traps could be used to create novel spatial probes and sensors. Inorganic nanowires have diameters substantially below the wavelength of visible light and have electronic and optical properties ${ }^{9,10}$ that make them ideal for subwavelength laser and imaging technology. Here we report the development of an electrode-free, continuously tunable coherent visible light source compatible with physiological environments, from individual potassium niobate $\left(\mathrm{KNbO}_{3}\right)$ nanowires. These wires exhibit efficient second harmonic generation, and act as frequency converters, allowing the local synthesis of a wide range of colours via sum and difference frequency generation. We use this tunable nanometric light source to implement a novel form of subwavelength microscopy, in which an infrared laser is used to optically trap and scan a nanowire over a sample, suggesting a wide range of potential applications in physics, chemistry, materials science and biology.

Nanometre-scale photonics is emerging as a key ingredient in novel sensing and imaging applications, as well as for advanced information technology, cryptography, and signal processing circuits. A versatile and useful nonlinear circuit element for integrated optical networks must be able to double the frequency of light using second harmonic generation (SHG), a second-order nonlinear optical phenomenon. In this process, two photons with the fundamental angular frequency $\omega_{1}$ are converted through a nonlinear crystal polarization into a single photon $\omega_{2}$ at twice the fundamental frequency $\left(\omega_{2}=2 \omega_{1}\right)$. We have recently demonstrated and characterized harmonic generation ${ }^{11}$, waveguiding, and optically pumped lasing in single nanowires of zinc oxide $^{12}$ and gallium nitride ${ }^{13}$. Despite the growing availability of building blocks such as light-emitting diodes ${ }^{10,14}$ lasers $^{13,15,16}$, photodetectors $^{17}$, and waveguides ${ }^{18}$, the field still lacks sufficiently small devices that efficiently generate tunable coherent photons. Here we show that the large second-order susceptibility $\chi^{(2)}$ of $\mathrm{KNbO}_{3}$ nanowires facilitates the generation of tunable, coherent visible radiation that is sufficient for in situ scanning and fluorescence microscopy.

We chose the perovskite oxide $\mathrm{KNbO}_{3}$ as the nanowire material because of its low toxicity, chemical stability, large effective nonlinear optical coefficients $\left(d_{\mathrm{eff}}=10.8-27 \mathrm{pm} \mathrm{V}^{-1}\right.$ at $\left.\lambda=1,064 \mathrm{~nm}\right)$ at room temperature $(298 \mathrm{~K})^{19}$, large refractive indices $(n=2.1-2.5)^{20}$, as well as its transparency in a wide range of wavelengths including the visible spectral region ${ }^{21}$. Single-crystalline $\mathrm{KNbO}_{3}$ nanowires were synthesized using a hydrothermal method ${ }^{22}$, and characterized as orthorhombic phase $(A m m 2)$ with the growth axis parallel to the [011] direction (Fig. 1a-e): the polar $c$ axis $^{23}$ is therefore $45^{\circ}$ off the nanowire's growth axis.

The $\mathrm{SHG}$ response of single $\mathrm{KNbO}_{3}$ nanowires were characterized first using femtosecond pulses described elsewhere ${ }^{24}$ and compared with measurements of $\mathrm{ZnO}$ nanowires which have been studied previously ${ }^{11}$. The nanowires were supported on amorphous silica coverslips and aligned such that the growth axis was orthogonal to the pump beam. The maximum SHG signals for both $\mathrm{KNbO}_{3}$ and $\mathrm{ZnO}$ nanowires $(\lambda=502 \mathrm{~nm})$ are shown in Fig. 1f, generated by introducing the fundamental beam $\left(\lambda=1,004 \mathrm{~nm}, 18 \mathrm{~kW} \mathrm{~cm}^{-2}\right)$. A direct comparison of the SHG signal collected from single $\mathrm{KNbO}_{3}$ and $\mathrm{ZnO}$ nanowires is complicated by the anisotropic scattering related to their respective rectangular and hexagonal cross-sections. However, a rough estimate for the $d_{\text {eff }}$ of $\mathrm{KNbO}_{3}$ nanowires based on the relative ratio of integrated signals is possible and we found it to be $\sim 9.1 \mathrm{pm} \mathrm{V}^{-1}$. This illustrates that the nonlinear polarizability of $\mathrm{KNbO}_{3}$ nanowires is larger than that for $\mathrm{ZnO}$, as expected from the consideration of bulk values.

The second key requirement for a versatile nonlinear circuit element for use in nano-photonics is wave mixing, specifically sum frequency generation (SFG) $\left(\omega_{3}=\omega_{1}+\omega_{2}\right)$ and difference frequency generation $\left(\omega_{3}=\left|\omega_{1}-\omega_{2}\right|\right)$. Fig. $1 \mathrm{~g}$ shows SFG signals $(\lambda=423 \mathrm{~nm}, 454 \mathrm{~nm})$ and SHG signals $(\lambda=525 \mathrm{~nm}, 700 \mathrm{~nm})$, obtained from a single $\mathrm{KNbO}_{3}$ nanowire by introducing fundamental beams at a variety of different frequencies via the tunable femtosecond pump. This demonstrates the ability of nanowire frequency converters to create four different waves from two fundamental input frequencies $\omega_{1}$ and $\omega_{2}: 2 \omega_{1}, 2 \omega_{2}$, and $\left|\omega_{1} \pm \omega_{2}\right|$. We did not observe the difference frequency generation signal corresponding to the SFG at $423 \mathrm{~nm}$ here because the expected wavelength $(\lambda=7,200 \mathrm{~nm})$ is outside current instrumental limits. The SHG signal at $400 \mathrm{~nm}$ was weak, owing to photoabsorption within the nanowire. This set of experiments demonstrates the ability of $\mathrm{KNbO}_{3}$ nanowires to generate continuously tunable and coherent light throughout the visible spectrum via nonlinear wave mixing. This capability, as well as the nanowire's subwavelength cross-section, enables the development of a novel form of scanning light microscopy.

Recently, laser trapping was used to optically manipulate nanowires in closed aqueous chambers ${ }^{8,25}$. We hypothesized that a single $\mathrm{KNbO}_{3}$ nanowire may, when optically trapped, be able to double the frequency of the trapping light and then waveguide this locally generated light to its ends. Single $\mathrm{KNbO}_{3}$ nanowires were optically trapped using a home-built infrared ${ }^{8}$ optical tweezers instrument (Fig. 2a) with the trap wavelength at 1,064 $\mathrm{nm}$, a wavelength popular for optical trapping of wet samples owing to the tolerance of living cells to infrared laser irradiation $^{26}$. We used an electron-multiplying charge-coupled device (CCD) to search for visible light radiating from trapped nanowires. 

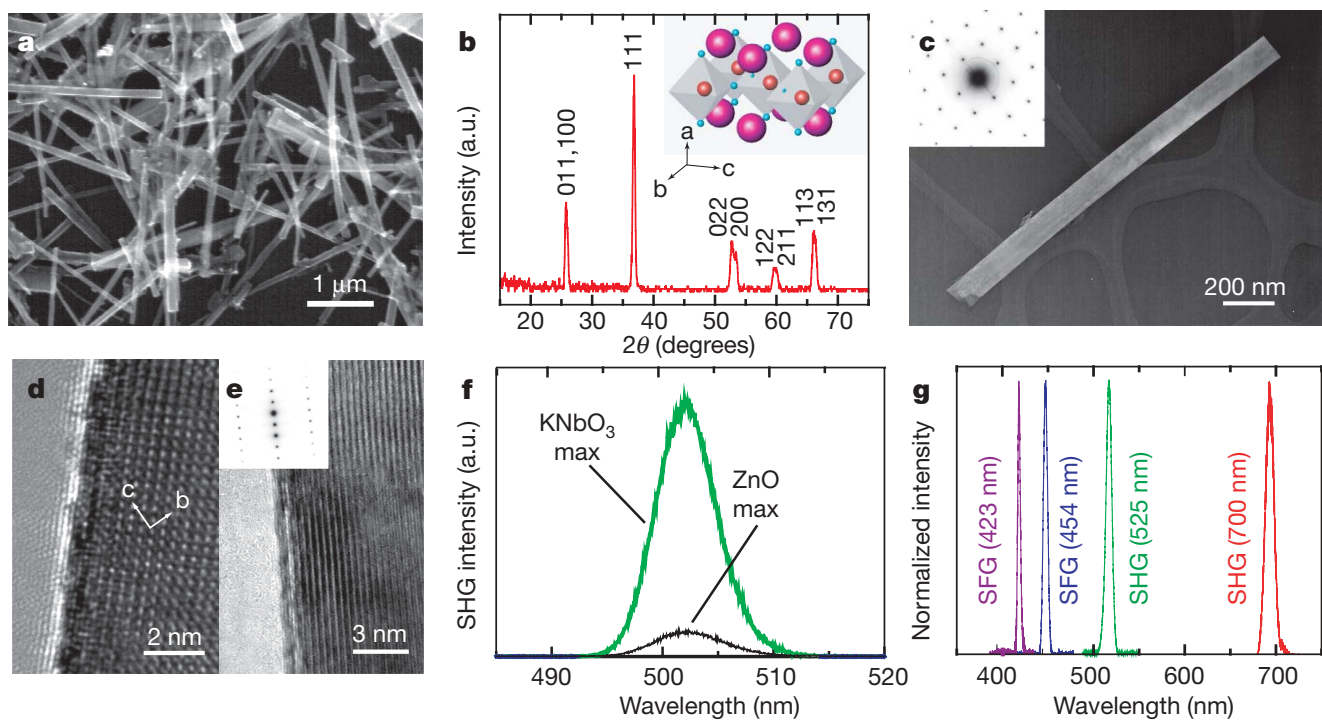

Figure $1 \mid \mathrm{KNbO}_{3}$ nanowires and their structural analysis. a, Scanning electron microscopy image of $\mathrm{KNbO}_{3}$ nanowires. $\mathbf{b}$, X-ray powder diffraction pattern of $\mathrm{KNbO}_{3}$ nanowires. The inset shows the unit cell structure of this material, with spontaneous polarization parallel to the $c$-axis. (a.u., arbitrary units.) c, Transmission electron microscope image of a $\mathrm{KNbO}_{3}$ nanowire and its electron diffraction pattern (inset) with [100] zone

As we hoped, light was observed to radiate from the distal end of trapped $\mathrm{KNbO}_{3}$ nanowires. We charted the radiation profile as a function of position along the nanowire's long axis by coarsely changing the focus of the top objective mounted on a micrometre stage (Fig. 2b). A diffraction-limited spot was observed at the distal end of the wire, revealing optical waveguiding away from the site of photon conversion and emission from the aperture defined by the nanowire's cross-section. Measurements with a colour CCD camera

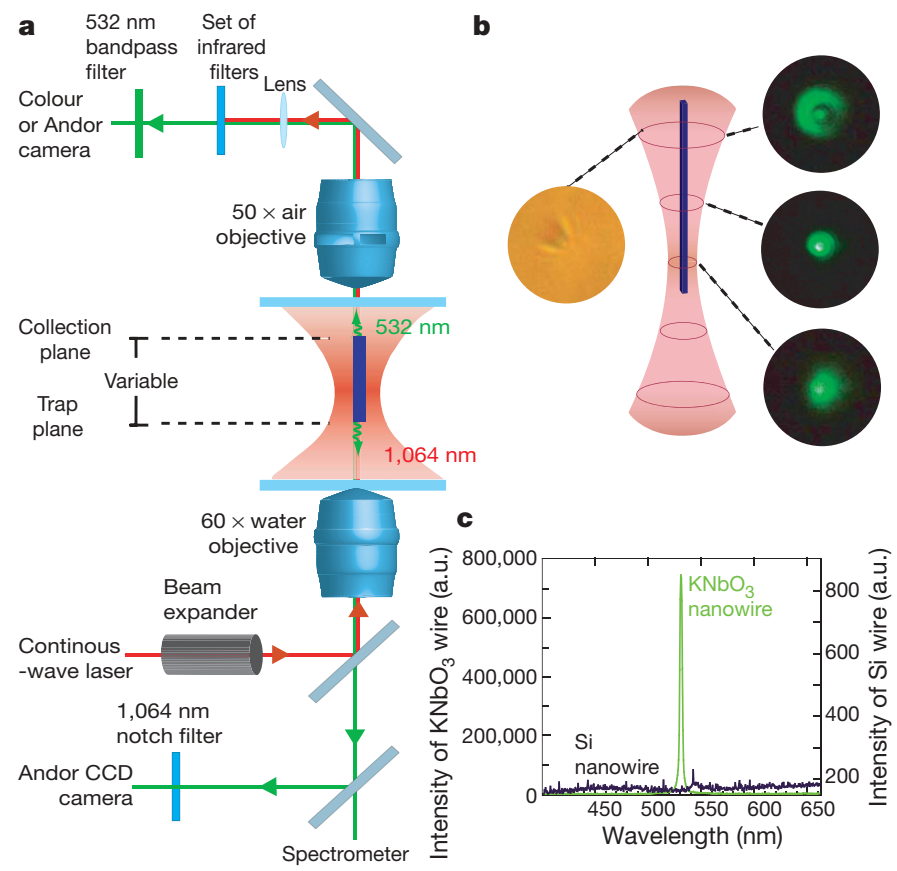

Figure 2 | Radiation from optically trapped single $\mathrm{KNbO}_{3}$ nanowires.

a, Detailed set-up for the single-beam optical trapping instrument. b, Bright field (left) and SHG (right) images of the trapped $\mathrm{KNbO}_{3}$ nanowire.

Waveguiding of the SHG signal (green) leads to diffraction rings at the distal (top) end of the nanowire which acts as a subwavelength aperture.

c, Observed spectra for $\mathrm{KNbO}_{3}$ and Si nanowires. Strong SHG signal at $\lambda=532 \mathrm{~nm}$ is collected from the trapped $\mathrm{KNbO}_{3}$ nanowire (green, left axis), while no signal was observed from Si nanowires (black, right axis). axis and [011] growth direction. d, e, High-resolution TEM images of single [011] growth-direction $\mathrm{KNbO}_{3}$ nanowires and an electron diffraction pattern (e, inset) with the zone axis of [100] (d) and [2-33] (e). f, Maximum SHG spectra of single $\mathrm{KNbO}_{3}$ and $\mathrm{ZnO}$ nanowires reflecting the larger nonlinear polarizibality of $\mathrm{KNbO}_{3}$. g, Panchromatic wavelengths generated by the nonlinear optical processes within individual $\mathrm{KNbO}_{3}$ nanowires.

show that SHG output from the nanowire varies less than $0.2 \%$ from the mean over $20 \mathrm{~s}$ of collection (Supplementary Fig. 3a).

The light emitted from the nanowire's end was collected through the lower trapping objective and spectrally analysed. Spectral analysis revealed that the light was green, with a wavelength of $531 \pm 1.8 \mathrm{~nm}$ (Fig. 2c), matching well with the expected SHG signal given a trapping/pump wavelength of $1,064 \mathrm{~nm}$. As a control, we also trapped $\mathrm{ZnO}$ and Si nanowires. $\mathrm{ZnO}$ (not shown) and Si nanowires (Fig. 2c, black line) did not produce visible light, supporting our conclusion that the green light from the $\mathrm{KNbO}_{3}$ nanowires was light generated inside the nanowire by SHG and indicating negligible SHG from symmetry breaking at the wire-water interface.

Assuming a non-depleted plane-wave pump geometry (Supplementary Information) and a typical trap irradiance of $\sim 10^{8} \mathrm{~W} \mathrm{~cm}^{-2}$, we calculate the total two-photon conversion efficiency $\eta_{2 \omega}$ to be at least $10^{-5}$. Estimations from this simple theoretical model are in agreement with second harmonic output powers of $\sim 10 \mathrm{nW}$ measured with the electron multiplying CCD (Supplementary Information). Although we used a $1,064 \mathrm{~nm}$ laser, there is no theoretical limitation to extending both trapping and SHG to the range of wavelengths demonstrated above with femtosecond pulses.

Unlike other nonlinear optical geometries in which alignment is accomplished manually via transducers, here the nonlinear crystal (the nanowire) spontaneously orients itself to the optical axis of the trap/pump laser, resulting in detectable SHG signal along the [011] growth axis. This favourable index matching allows the entire nanowire cavity to participate in the production of second harmonic photons. It is possible that index matching could be further improved by controlling the temperature of the buffer, although we did not explore this here.

Having in hand a nanometric, raster-scannable source of coherent visible light, we wondered whether it could be used to image objects. We used a simple transmission geometry analogous to near-field scanning optical microscopy (NSOM) $)^{27,28}$, in which the sample is scanned through a beam, modulating the fraction of light arriving at a detector. The ultimate resolution of such a transmission microscope depends on the radiation characteristics of the illumination source. In this approach the resolution is of the order of the crosssection of the illumination aperture but many other factors are also important, such as the quality of the probe's end-facets, far-field collection optics and implemented feedback control. 
a

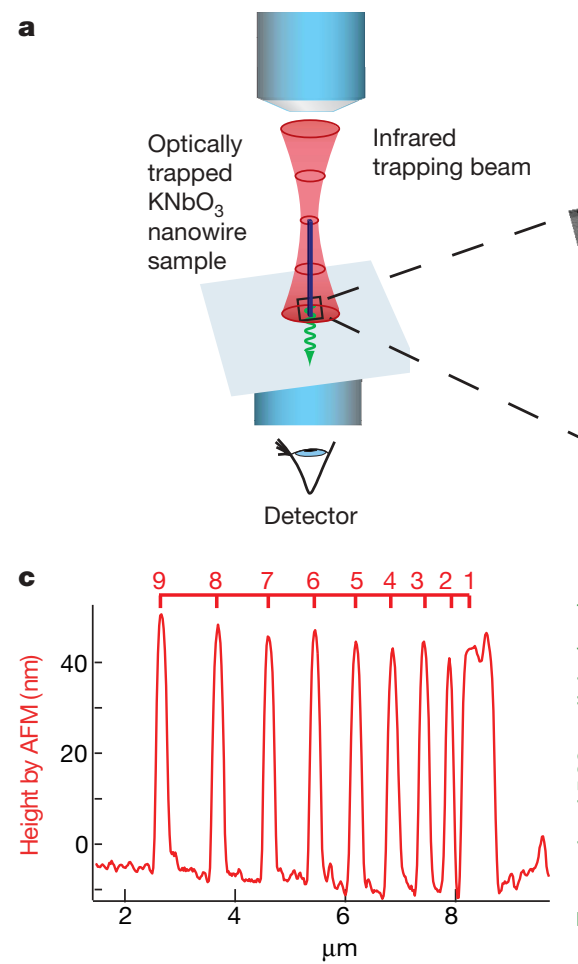

b
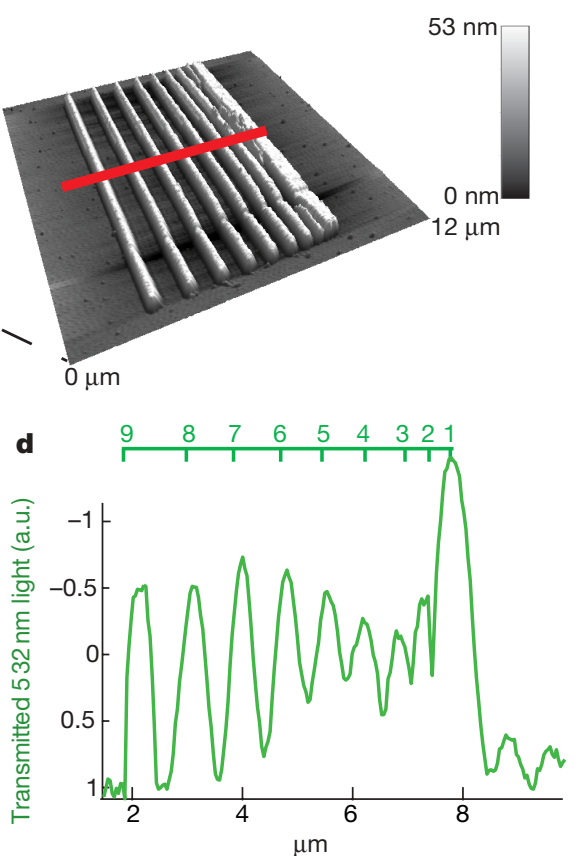

Figure 3 | Transmission line scan of metallic surface pattern with laser trapped $\mathrm{KNbO}_{3}$ nanowire. a, Schematic of inverted optical scanning configuration. b, AFM topographic image of thermally evaporated pattern of gold stripes on a glass coverslip. c, AFM line scan from region indicated in $\mathbf{b}$. d, Optical transmission profile captured by scanning a single $\mathrm{KNbO}_{3}$ nanowire over the metallic surface structure. The nanowire used to create the transmission line scan was measured by AFM to have these dimensions: width $=122 \mathrm{~nm}$, length $=1.4 \mu \mathrm{m}$ and height $=53 \mathrm{~nm}$. The peak index (there are nine lines in total being measured) is shown at the top of $\mathbf{c}$ and $\mathbf{d}$.
To see whether radiating nanowires can be used to image objects and to characterize the resolution of such a 'nanowire scanning microscopy' (Fig. 3a), we fabricated a test pattern via electron beam lithography consisting of a series of 200-nm-wide, 50-nm-thick lines

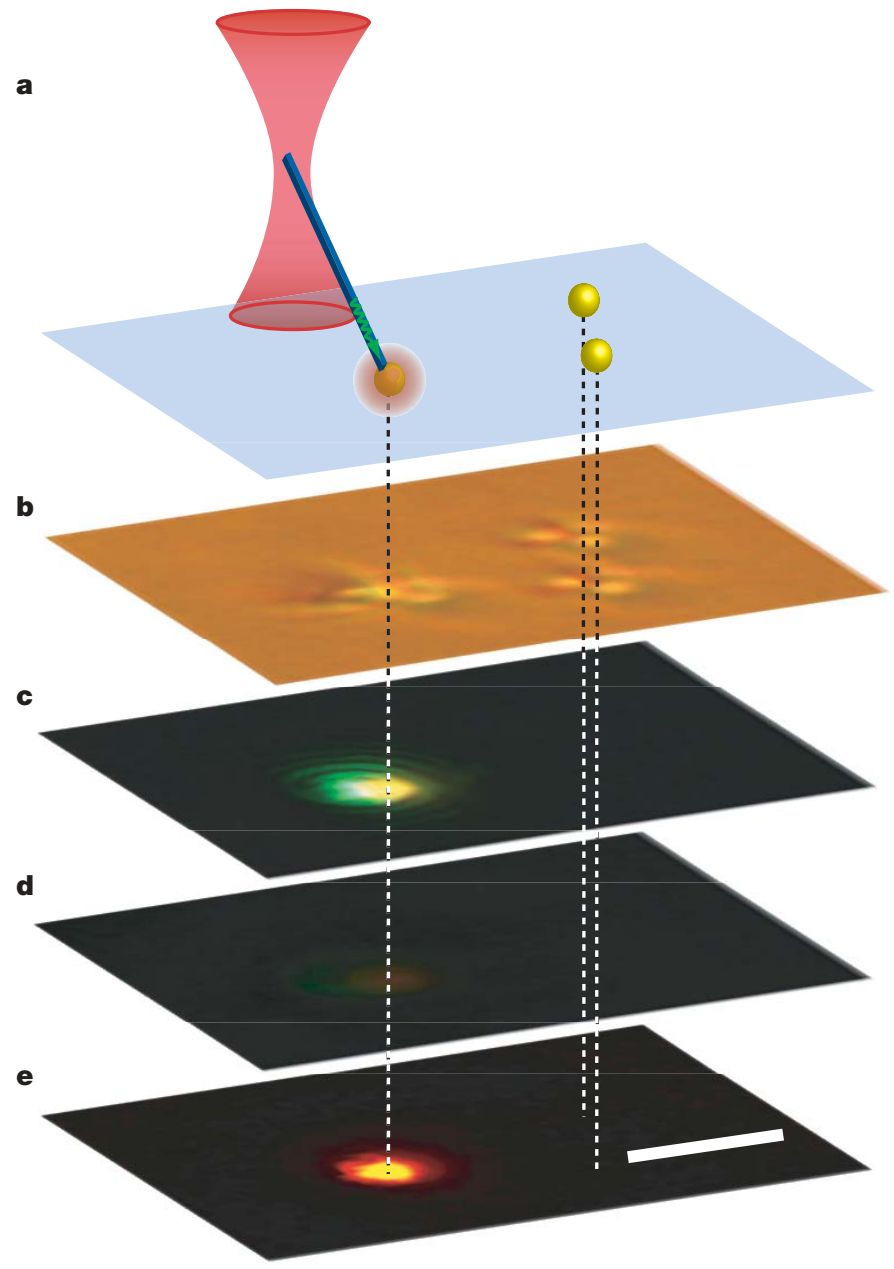

of gold on transparent glass coverslips with decreasing pitch between each line. A 'benchmark' image of the gold pattern was obtained by atomic force microscopy (AFM; Fig. 3b) and shows the progressively finer separation between each line, decreasing from $\sim 1,000$ to $\sim 200 \mathrm{~nm}$ (Fig. 3c).

The laser trap was then used to raise a single $\mathrm{KNbO}_{3}$ nanowire to the top coverslip surface with the gold pattern while a piezoelectric stage with nanometre positioning resolution was used to scan the pattern with respect to the wire. Each time the trapped wire passed over a gold feature there was a reduction of transmitted second harmonic emission. Scanning the nanowire tip directly across the surface resulted in a transmitted intensity map with local spatial resolution of the order of the wire's diameter (Fig. 3d). Measurements of the pitch between lines within the gold pattern were made for both AFM and nanowire optical transmission line scans and agreed to within $\sim 10 \%$ (Supplementary Table 1). As expected, the minimum resolvable feature-size (pitch) decreases as the crosssection of the nanowire-probe decreases (Supplementary Fig. 2c, f).

The overall resolution in these experiments is reduced by the use of far-field optics for image collection and (presumably) the fluctuations of the nanowire in the optical potential. Root-mean-square lateral displacements of the wire owing to thermal fluctuations in the optical potential are estimated to be $\sim 10 \mathrm{~nm}$ by measurement of the power spectrum of a trapped wire using a position-sensitive photodetector $^{8}$ (Supplementary Fig. 1d); longitudinal fluctuations will be larger owing to reduced confinement of the wire along the optical axis. Indeed, one of the most urgent next steps in the development of nanowire scanning microscopy will be to find ways of reducing longitudinal fluctuations; a theoretically ideal probe would be tapered, because this would increase optical confinement and also the crystal volume in the region of largest field intensity.

Figure 4 | POPO-3 bead excitation by waveguided SHG signal from an optically trapped $\mathrm{KNbO}_{3}$ nanowire. a, Inverted schematic drawing of the experimental configuration. $\mathbf{b}$, Bright-field optical image of the beads, with the nanowire in contact with the leftmost bead. c, Colour CCD fluorescence image of $\boldsymbol{b}$, showing green light emission from the nanowire and the orange emission from the bead. $d$, Control image of the same beads with infrared radiation in the plane from $b$, but without a trapped nanowire. e, Digital subtraction of $\mathbf{d}$ from c. Scale bar, $3 \mu \mathrm{m}$. 
In addition to exploring this scanning transmission mode, it is also possible to investigate this new scanning probe in a fluorescence mode. We optically trapped a single $\mathrm{KNbO}_{3}$ nanowire and touched its distal end to a fluorescent bead (Fig. 4a). In some instances, wires adhered to the bead at the contact point presumably owing to the bead's carboxylate surface functionalization (for adhesion to the top coverslip). Excitation occurred in this near-field geometry that generated a distinct orange fluorescence at the contact point (Fig. 4c). Removal of the nanowire reduced orange emission from the bead by more than 80 fold (Fig. 4d), indicating that the $532 \mathrm{~nm} \mathrm{SHG} \mathrm{emitted} \mathrm{by} \mathrm{the} \mathrm{nanowire}$ was the predominant source of excitation relative to two-photon fluorescence. Figure 4e shows the intensity difference between Fig. 4c and $\mathrm{d}$, obtained by digital subtraction of the red components of the images, which displays the distinctive fluorescence emission due to SHG excitation from the optically trapped $\mathrm{KNbO}_{3}$ nanowire.

The next step in the development of nanowire-scanning microscopy will be to adapt the now highly refined signal-processing and signal-deconvolution algorithms originally developed for AFM and NSOM to this new method. Although already providing promising lateral resolution (Fig. 3), the utility of nanowire scanning microscopy will further increase once it is understood how to convolve and best interpret the various readouts (fraction of excitation light transmitted, sample fluorescence emission), and the mechanical deflection of the nanowire as it is tapped over a sample, as measured from scattering between the trapping laser ${ }^{29,30}$ and the nanowire. Similar to NSOM, nanowire scanning microscopy is an inherently mechano-optical form of microscopy because the mechanical probe (the nanowire) also functions as a source of coherent visible radiation. One potential advantage of nanowire scanning microscopy is its possible extension to massively parallel excitation arrays using holographic optical elements ${ }^{25}$ that would be compatible with sealed environments, such as microfluidic chambers.

\section{METHODS SUMMARY}

$\mathrm{KNbO}_{3}$ nanowires were synthesized using a hydrothermal method ${ }^{22}$. For nonlinear wave mixing measurements, SFG spectra (Fig. 1f, g) were collected by introducing two fundamental beams with the same polarization directions ${ }^{11}$. Nanowire optical trapping was performed according to ref. 8. The continuous-wave infrared laser $(\lambda=1064 \mathrm{~nm}, \sim 1 \mathrm{~W})$ was introduced from the bottom side of the chamber to trap the nanowire as well as to generate the second harmonic wave at $\lambda=532 \mathrm{~nm}$. The trapping instrument was modified with a colour CCD (Fig. 2a) to acquire images of optically trapped $\mathrm{KNbO}_{3}$ wires at various focal planes by moving the top objective along the optical axis without translation of the trapping point. Spectra were taken through the bottom objective. Gold surface-patterns were made for the scanning measurements using glass coverslips coated with a $2 \mathrm{~nm}$ chromium bonding layer. Electron beam lithography was used to define $\sim 200$-nm-wide lines with variable pitch. Thermal evaporation of gold yielded lines of gold $\sim 50 \mathrm{~nm}$ thick. The transmitted SHG signal was collected with a high-speed electron-multiplying CCD camera (Andor, iXon), and custom software written in $\mathrm{C}++$ was used to control the scan size and speed of the $x y z$-piezo stage. Fluorescent polystyrene beads containing POPO-3 dye molecules were fixed to the surface of a glass coverslip via carboxylate surface functionalization. The distal end of the trapped nanowire was brought in contact with one of the beads (Fig. $4 \mathrm{a}-\mathrm{c}$ ) and colour CCD images were taken (Photometrics, CoolSNAPcf).

Full Methods and any associated references are available in the online version of the paper at www.nature.com/nature.

\section{Received 20 October 2006; accepted 11 May 2007.}

1. Sanchez, E. J., Novotny, L. \& Xie, X. S. Near-field fluorescence microscopy based on two-photon excitation with metal tips. Phys. Rev. Lett. 82, 4014-4017 (1999)

2. Inouye, Y. \& Kawata, S. Near-field scanning optical microscope with a metallic probe tip. Opt. Lett. 19, 159-161 (1994).

3. Donnert, G. et al. Macromolecular-scale resolution in biological fluorescence microscopy. Proc. Natl Acad. Sci. USA 103, 11440-11445 (2006).

4. Gustafsson, M. G. L. Nonlinear structured-illumination microscopy: Wide-field fluorescence imaging with theoretically unlimited resolution. Proc. Natl Acad. Sci. USA 102, 13081-13086 (2005).
5. Betzig, E. et al. Imaging intracellular fluorescent proteins at nanometer resolution. Science 313, 1642-1645 (2006).

6. Ghislain, L. P. \& Webb, W. W. Scanning-force microscope based on an optical trap. Opt. Lett. 18, 1678-1680 (1993).

7. Florin, E. L. Pralle, A. Horber, J. K. H. \& Stelzer, E. H. K. Photonic force microscope based on optical tweezers and two-photon excitation for biological applications. J. Struct. Biol. 119, 202-211 (1997).

8. Pauzauskie, P. J. et al. Optical trapping and integration of semiconductor nanowire assemblies in water. Nature Mater. 5, 97-101 (2006).

9. Yang, P. The chemistry and physics of semiconductor nanowires. MRS Bull. 30, 85-91 (2005).

10. Sirbuly, D. J., Law, M., Yan, H. Q. \& Yang, P. D. Semiconductor nanowires for subwavelength photonics integration. J. Phys. Chem. B 109, 15190-15213 (2005)

11. Johnson, J. C. et al. Near-field imaging of nonlinear optical mixing in single zinc oxide nanowires. Nano Lett. 2, 279-283 (2002)

12. Johnson, J. C., Yan, H. Q., Yang, P. D. \& Saykally, R. J. Optical cavity effects in ZnO nanowire lasers and waveguides. J. Phys. Chem. B 107, 8816-8828 (2003).

13. Pauzauskie, P. J., Sirbuly, D. J. \& Yang, P. D. Semiconductor nanowire ring resonator laser. Phys. Rev. Lett. 96, 14903 (2006).

14. Qian, F. et al. Gallium nitride-based nanowire radial heterostructures for nanophotonics. Nano Lett. 4, 1975-1979 (2004).

15. Huang, Y., Duan, X., Wei, Q. \& Lieber, C. M. Directed assembly of onedimensional nanostructures into functional networks. Science 291, 630-633 (2001).

16. Duan, X. F., Huang, Y., Agarwal, R. \& Lieber, C. M. Single-nanowire electrically driven lasers. Nature 421, 241-245 (2003).

17. Kind, H., Yan, H. Q., Messer, B., Law, M. \& Yang, P. D. Nanowire ultraviolet photodetectors and optical switches. Adv. Mater. 14, 158-160 (2002).

18. Law, M. et al. Nanoribbon waveguides for subwavelength photonics integration. Science 305, 1269-1273 (2004)

19. Shoji, I., Kondo, T., Kitamoto, A., Shirane, M. \& Ito, R. Absolute scale of secondorder nonlinear-optical coefficients. J. Opt. Soc. Am. B 14, 2268-2294 (1997)

20. Zysset, B., Biaggio, I. \& Gunter, P. Refractive indexes of orthorhombic $\mathrm{KNbO}_{3} .1$. Dispersion and temperature-dependence. J. Opt. Soc. Am. B 9, 380-386 (1992).

21. Kudo, K., Kakiuchi, K., Mizutani, K. \& Fukami, T. Characterization of $\mathrm{KNbO}_{3}$ crystal by traveling solvent floating zone (TSFZ) method. Jpn. J.Appl. Phys. Part 142, 6099-6101 (2003).

22. Magrez, A. et al. Growth of single-crystalline $\mathrm{KNbO}_{3}$ nanostructures. J. Phys. Chem. B 110, 58-61 (2006)

23. Biaggio, I., Kerkoc, P., Wu, L. S., Gunter, P. \& Zysset, B. Refractive indexes of orthorhombic $\mathrm{KNbO}_{3}$. 2. Phase-matching configurations for nonlinear-optical interactions. J. Opt. Soc. Am. B 9, 507-517 (1992).

24. Knutsen, K. P., Messer, B. M., Onorato, R. M. \& Saykally, R. J. Chirped coherent anti-Stokes Raman scattering for high spectral resolution spectroscopy and chemically selective imaging. J. Phys. Chem. B 110, 5854-5864 (2006).

25. Agarwal, R. et al. Manipulation and assembly of nanowires with holographic optical traps. Opt. Express 13, 8906-8912 (2005).

26. Ashkin, A., Dziedzic, J. M. \& Yamane, T. Optical trapping and manipulation of single cells using infrared-laser beams. Nature 330, 769-771 (1987).

27. Pohl, D. W., Denk, W. \& Lanz, M. Optical stethoscopy-image recording with resolution lambda/20. Appl. Phys. Lett. 44, 651-653 (1984).

28. Betzig, E., Trautman, J. K., Harris, T. D., Weiner, J. S. \& Kostelak, R. L. Breaking the diffraction barrier - optical microscopy on a nanometric scale. Science 251, 1468-1470 (1991).

29. Florin, E.-L., Pralle, A., Stelzer, E. H. K. \& Hörber, J. K. H. Photonic force microscope calibration by thermal noise analysis. Appl. Phys. A 66, 75-78 (1998).

30. Grier, D. G. A revolution in optical manipulation. Nature 424, 810-816 (2003).

Supplementary Information is linked to the online version of the paper at www.nature.com/nature.

Acknowledgements This work was supported in part by the Dreyfus Foundation and the US Department of Energy (P.Y.), the University of California, Berkeley

(J.L.), the Experimental Physical Chemistry Program of the National Science Foundation, and the NASA SRLDA program (R.M.O. and R.J.S.). Y.N. thanks SONY for a research fellowship and P.J.P. thanks the NSF for a graduate research fellowship. Work at the Lawrence Berkeley National Laboratory was supported by the Office of Science, Basic Energy Sciences, Division of Materials Science of the US Department of Energy. We thank T. Kuykendall for transmission electron microscope observations and the National Center for Electron Microscopy for the use of their facilities, L. Sohn for AFM facilities, N. Switz for comments on the manuscript and W. Liang for microfabrication of gold patterns.

Author Contributions Y.N. performed the synthesis and structural characterization of the $\mathrm{KNbO}_{3}$ wires. Y.N. and R.M.O. designed, performed and analysed the wave mixing experiment. P.J.P. and A.R. designed, performed and analysed the laser trapping and nanoprobe imaging experiments.

Author Information Reprints and permissions information is available at www.nature.com/reprints. The authors declare no competing financial interests. Correspondence and requests for materials should be addressed to J.L. (liphardt@physics.berkeley.edu) or P.Y. (p_yang@berkeley.edu). 


\section{METHODS}

Materials preparation. Potassium hydroxide and niobium pentoxide were mixed with deionized water for $2 \mathrm{~h}$ at room temperature ${ }^{22}$. The slurry was transferred to Teflon vessels and then heated at $150^{\circ} \mathrm{C}$ for $2-6$ days using the stainless autoclaves. Sizes could be varied by adjusting the reaction time. Scanning electron microscopy images showed that the products are collections of rectangularly shaped nanowires with widths ranging from 40 to $400 \mathrm{~nm}$ and the lengths from 1 to $20 \mu \mathrm{m}$ (Fig. 1a). The products were identified as the single-phase orthorhombic $\mathrm{KNbO}_{3}(A m m 2$, $a=0.3984 \mathrm{~nm}, b=0.5676 \mathrm{~nm}$ and $c=0.5697 \mathrm{~nm}$ ) from X-ray powder diffraction measurements (Fig. 1b). Transmission electron microscope images and electron diffraction measurements (Fig. 1c-e) show that the nanowires grow parallel to [011] directions. Twinned structures were observed in some nanowires.

Nonlinear optical measurements. Single nanowire suspensions were obtained after sonication in iso-propanol and placed on a transparent glass coverslip with a nanomanipulator. Femtosecond pulses generated by a regeneratively amplified Ti:sapphire oscillator (wavelength: $\lambda=800 \mathrm{~nm}, 90 \mathrm{fs}, 1 \mathrm{kHz}$ ) were used to pump an optical parametric amplifier where the wavelength is continuously tunable between 1,150-2,600 $\mathrm{nm}$ and can be doubled in frequency by a $\mathrm{BaB}_{2} \mathrm{O}_{4}$ crystal outside the optical parametric amplifier, providing access to shorter wavelengths before being introduced to the nanowire perpendicular to its growth direction (Fig. 1f, g). The beam spot size was about $1 \mu \mathrm{m}$, which is larger than the width of the wires but smaller than their lengths. SFG spectra were measured by introducing two fundamental beams with the same polarization directions. Nanowire output with $\lambda=423 \mathrm{~nm}$ was produced as a result of SFG: $(423 \mathrm{~nm})^{-1}=(800 \mathrm{~nm})^{-1}+$ $(900 \mathrm{~nm})^{-1}$, and other spectra were obtained as SFG: $(454 \mathrm{~nm})^{-1}=(800 \mathrm{~nm})^{-1}+$ $(1,050 \mathrm{~nm})^{-1}$, SHG: $(525 \mathrm{~nm})^{-1}=2 \times(1,050 \mathrm{~nm})^{-1}$ and $(700 \mathrm{~nm})^{-1}=2 \times$ $(1,400 \mathrm{~nm})^{-1}$. From the experimental configuration, coefficients are identified as $d_{\text {eff }}=d_{31}$ for the $\mathrm{KNbO}_{3}$ nanowire, and $d_{\mathrm{eff}}=d_{33}$ for the $\mathrm{ZnO}$ nanowire.

Optical trapping. Nanowires were dispersed into deionized water inside the sample chamber ${ }^{3}$. The continuous-wave infrared laser $(\lambda=1,064 \mathrm{~nm}, \sim 1 \mathrm{~W})$ was introduced from the bottom side of the chamber to trap the nanowire as well as to generate the second harmonic wave at $\lambda=532 \mathrm{~nm}$. The chamber can be moved coarsely with a manual stage, and finely with a three-axis piezostage. Colour CCD images are taken at various focal planes by moving the top objective along the optical axis without translation of the trapping point, whereas spectra were taken through the bottom objective. On the basis of the experimental configuration and material properties, the nonlinear optical coefficients are considered to be $d_{\text {eff }}=0$ for both $\mathrm{ZnO}$ and Si nanowires. The SHG signal from surface inversion symmetry breaking was too small to detect in this study.

Nanowire scanning. Gold line patterns were fabricated using electron beam lithography with glass coverslips coated with a $2 \mathrm{~nm}$ chromium bonding-layer. The electron beam was used to define $\sim 200$-nm-wide line patterns with variable pitch. After $50 \mathrm{~nm}$ of thermal gold deposition, reactive ion etching was used to remove residual chromium from glass to prevent laser heating and bubble formation during scanning measurements. Transmitted SHG signal was collected (at a frame rate of $20 \mathrm{~Hz}$ ) by using an Andor iXon camera. Custom software written in $\mathrm{C}++$ was used to control the scan size and speed of $x y z$-piezo stage (Nano-UHV100; Mad City Lab). During raster scanning experiments the microfabricated gold lines modulated the SHG signal transmitted through the coverslip owing to a change of the local optical transparency. Future work to improve feedback during raster scanning might employ high-speed analysis of the variability in SHG output due to changes in phase matching from opto-mechanical deflections of the nanowire. All scanning data sets were processed using custom software Igor Pro 5 (Wavemetrics). After scanning, wires were fixed to the glass surface using non-specific binding. An AFM (Asylum Research MFP3D combined with Nikon inverted microscope) operating in tapping mode was used to image fixed nanowires used in the scanning measurements as well as electron beam substrates following reactive ion etching. All AFM images were obtained with the MikroMasch probes (model number NSC36/ $\mathrm{NoAl}$ ) having a nominal spring constant $0.6 \mathrm{~N} \mathrm{~m}^{-1}$.

Bead excitation. Polystyrene beads ( $1 \mu \mathrm{m}$ in diameter) containing the fluorescent dye POPO-3 (main emission peak $\sim 570 \mathrm{~nm}$, absorption peak $\sim 532 \mathrm{~nm}$, Molecular Probes, Fig. 4) were fixed to the surface of a glass coverslip via carboxylate surface functionalization. The distal end of the trapped nanowire is brought in contact with one of the beads (Fig. $4 \mathrm{a}-\mathrm{c}$ ) and colour CCD images were taken (Photometrics, CoolSNAPcf). The initial position was set using a computer-controlled piezoelectric stage. The stage could be actuated to remove the nanowire. Finally, the empty laser trap was moved to the original position for corresponding two-photon excitation (Fig. 4d). The subtraction of Fig. 4d from Fig. $4 \mathrm{c}$ is performed using the red component of the colour images. Spectra in Supplementary Fig. 4 were obtained (Acton, SpectraPro 300i) using a sample chamber filled with a 20 vol. \% aqueous POPO-3 dye solution. The luminescence spectrum of SHG-excitation was obtained by subtraction of pure two-photon luminescence (without trapping a nanowire) from spectra with a trapped $\mathrm{KNbO}_{3}$ nanowire. 